\title{
Parallel processing of the attributes of single stimuli*
}

\author{
ESTHER SARAGA $\dagger$ and TIM SHALLICE $\dagger \dagger$ \\ University College London, London, England
}

\begin{abstract}
Three experiments are described, in which Ss had to decide whether or not single stimuli, varying along the dimensions of shape and color, satisfied a given criterion. In different conditions, the number of dimensions relevant to the criterion was varied. The first experiment indicates that simple interpretations of a standard Nickerson procedure are potentially artifactual. In the last two experiments, a design involving different difficulties of discrimination was used so that a serial interpretation of the results could be convincingly rejected, even for unpracticed Ss. These results strongly support the view that perceptually parallel processing normally occurs in the identification of single multidimensional stimuli. By this interpretation, apparently similar tasks may produce different patterns of results because of the use of different decision processes by the Ss.
\end{abstract}

Research on serial and parallel processing in perception has developed considerably since it began in the early 1960s with the work of Neisser (1963) and Sternberg (1963). The idea of parallel (or multiple) processing of stimulus attributes received support from Neisser's visual search experiments with letters (Neisser, 1963), but his task was rather too complex for unequivocal conclusions to be drawn. Later Es have, therefore, attempted to test the theory using simpler situations and employing multidimensional stimuli.

Three experimental situations have been widely used to investigate the mode of processing multidimensional stimuli. One line of research, which stemmed from the experiment of Egeth (1966), uses a situation in which two stimuli differing on $0,1,2$, or 3 dimensions are presented simultaneously, and the $S$ must respond according to whether they are the same or different. The results of Egeth's experiment supported the serial model. In a variant of Egeth's situation, the two stimuli are presented successively for comparison. Nickerson (1967a) and Grill (1971) have both compared simultaneous and successive presentation of stimuli. Serial results were obtained for both methods of presentation, although Grill found that for the simultaneous presentation there was a gradual shift with practice from serial to parallel processing.

A third experimental method is the "stimulus categorization" method used by Nickerson (1967b). In this method, only one stimulus is presented and the S's response depends on whether or not the presented stimulus matches a target stimulus, which remains constant over a block of trials. Examples of targets used

*The experiments in this paper form part of research to be reported in a thesis to be submitted for the degree of $\mathrm{PhD}$ to London University by the first author under the supervision of the second author. The first author was supported by a Scholarship for Training in Research Methods from the Medical Research Council. We are grateful to $\mathbf{P}$. Dain for colorimetric advice.

$†$ Present address: Department of Sociology and Psychology, Chelsea College, London, S.W.3. England.

† Department of Psychology. National Hospital, London. W.C.1. England. are "red" and "large red square"; stimuli may differ from the target on $0,1,2$, or 3 dimensions. Nickerson obtained results which were equivocal with respect to the serial/parallel question. The results of Marcel (1969), using a similar technique, supported a serial model in the early stages of practice, but there was evidence of a change to parallel processing in later stages.

The general tendency is for these results to indicate serial processing at least in the early stages of practice. It is, however, in conflict with the results of other investigations (e.g., Hawkins, 1969; Biederman \& Checkosky, 1970; Downing \& Gossman, 1970), which, given somewhat more complex analyses, suggest parallel processing. The results of yet others (e.g., Nickerson, 1967b) do not support either.

This somewhat confused situation has led some theorists (e.g., Garner \& Felfoldy, 1970; Grill, 1971; Rabbitt, 1971) to criticize the assumption that there is a normal mode of information processing which can be determined experimentally to be either serial or parallel. Thus, some recent research (e.g., Grill, 1971) has been directed towards specifying those tasks and stimulus conditions under which the different modes of processing will occur.

While agreeing that the situation is, indeed, very complex, this type of position seems to the present authors in one way too optimistic, in another too pessimistic. To presuppose that clear criteria exist for differentiating between serial and parallel processes for any particular task and stimuli seems optimistic. It is pessimistic insofar as it is assumed that the normal mode of operation of any component process will not be found to be either parallel or serial. Our strategy, therefore, is to attempt to obtain more clear-cut criteria for assessing the serial/parallel question and to examine a particular component process-the operation of the perceptual mechanisms. Three specific points should clarify this position.

\section{Crudeness of Criteria}

A common procedure in this type of task has been to 
analyze the results in terms of the number of dimensions to which the $S$ has to attend on a given trial (the relevant dimensions) and the number of dimensions by which the stimulus differs from the target (Nickerson, 1967b, design) or by which the two stimuli differ (Egeth design). Nickerson calls the two numbers $r$ (for relevant) and $f$ (for fail the criterion). The argument given below will refer to the Nickerson design, but a complementary argument exists for the Egeth design. With the Nickerson design, it appears to be fairly simple to distinguish between serial and parallel models. Consider those stimuli which differ from the target on one dimension only $(f=1)$. We shall refer to this dimension as the "critical" dimension, since the correct decision ("no") must be based on the outcome of processing this dimension. If more than one dimension is relevant $(r>1)$ and Ss operate serially, they must sometimes process "noncritical" dimensions before the "critical" one. They cannot know in advance which dimension will be the critical one for the next stimulus. However, when only the critical dimension is relevant $(r=1)$, that dimension will presumably always be processed first. RTs will, therefore, be longer when more than one dimension is relevant. (In Nickerson's terminology, RT increases with $r$ for fixed $f$.) On the other hand, on an unlimited parallel model, when more than one dimension is relevant, simultaneous processing of the noncritical dimensions will not affect the time to process the critical one, so that RTs will be independent of the number of dimensions relevant. (In Nickerson's terminology, RT is independent of $r$ for fixed $f$.) However, this method of analysis involves averaging across stimuli with different critical dimensions, and it will be demonstrated in Experiment I that such averaging can lead to artifacts. A related argument will be given in the Discussion to provide an alternative explanation for the way the pattern of results changes with practice [normally supposed to represent a change from serial to parallel processing (Marcel, 1970; Grill, 1971)].

One factor which exacerbates the problem of assessing the fit between data and theory is the number of variants of parallel and serial models that have been suggested. Thus, Egeth (1966) describes four parallel and four serial models; Corcoran (1971) adds to this by including the idea of "limited-power" or "limited-capacity" parallel models, in which component processes are carried out simultaneously, at the expense of an overall reduction in speed (and possibly accuracy) of processing. [See also Egeth \& Pachella (1969) and Lindsay, Taylor, \& Forbes (1968) for similar parallel models.]

Also, the term "parallel" processing has not always been used in the same way; in particular, there is some confusion between the notions of "independent" and "parallel" processes. The work to be discussed here is entirely concerned with the simultaneity criterion of parallel processing. That is, it is assumed that on a self-terminating, unlimited, parallel model, two processes can be carried out as quickly as one. On the other hand. on a self-terminating serial model, two processes must take longer than one. We will consider mainly the self-terminating serial and the unlimited parallel models, since these provide the simplest explanations of much of the data in the literature. At certain points, it will be shown that exhaustive serial and "limited-capacity" parallel models do not fit our data.

\section{The Validity of E-Defined Dimensions}

Experimenters have normally assumed that the dimensions on which they conceive the stimuli to vary are the dimensions used by the $\mathrm{S}$. This assumption has recently been strongly criticized (Garner \& Felfoldy, 1970) on the ground that different stimulus dimensions produce different patterns of results. They argued that the investigation of the psychological status of dimensions must precede that of the serial or parallel processing of dimensions. However, the concept of "integrality," which they use, has been differently defined by different authors and, if it is eventually defined in terms of process, then the serial/parallel question will not be a secondary one. This will be discussed further later.

However, as Corcoran (1971) has pointed out, if one is concerned with the potentiality for parallel processing, it is inappropriate to use dimensions that may be collapsible into a single dimension, e.g., horizontal and vertical displacement of a point [Morton's (1969) "evidence-combination"]. In particular, Corcoran refers to the dimensions of brightness and size, the same dimensions used by Biederman and Checkosky (1970) in their demonstration of parallel processing, as potentially collapsible into one of total luminance. By contrast, it may also be inappropriate to use obscure or difficult dimensions, since they may require a different type of attentional process.

\section{Component Process Analysis of Performance}

It has been pointed out a number of times (e.g., Nickerson, 1967b; Garner \& Morton, 1969) that the serial/parallel question cannot be validly posed with respect to the task as a whole, but only with respect to the operation of particular component processes. In particular, it is often assumed (e.g., Nickerson, 1967b) that the decision as to whether the stimulus matches the target is not delayed after the completion of the necessary earlier component processes, so that the pattern of response times reflects the pattern of these processes. However, as Treisman (1969) points out. the RTs may also be determined by the decision process of selecting the correct response. This argument can be used to explain the findings of Nickerson (1967b) and Marcel (1969) that performance using disjunctively defined targets is serial for naive Ss. The instructions in a 
disjunctive task involve deciding, for example, whether a stimulus is "red" or "square," and it has been shown that such instructions produce cognitive complications (Trabasso, Rollins, \& Shaughnessy, 1971). The cognitive difficulty in this instruction may therefore lead to a set for the decision stage to operate serially, even though the attributes have been perceptually processed in parallel. Such an explanation is supported by the much greater number of errors made by unpracticed Ss in the disjunctive condition $(>30 \%)$ compared with the conjunctive condition (8\%) in Nickerson's experiment. (The values given are for the $r=3, f=1$ condition.)

If the component processes at one stage operate serially, then the overall performance will produce a serial pattern of results. On the other hand, a parallel pattern will be produced only if all component processes operate in parallel at every stage. It follows that if one is primarily interested in the potentiality for perceptual processing in parallel, conjunctively defined targets, which are cognitively simpler, are more appropriate.

Related arguments could be given for the serial aspects of the results of Lindsay and Lindsay's (1966) experiment, in which no response could ever be determined from the analysis of any single dimension.

Another example of the need to consider the different component processes comes when one compares the Nickerson (1967b) and Egeth (1966) designs. If one is concerned with the processes involved in the identification of a single stimulus, the simultaneous comparison method of Egeth (1966) does not seem as appropriate, for reasons given by Treisman (1969). The primary defect is that the problem of serial or parallel processing of the attributes of a single stimulus is confused with that of serial or parallel processing over spatial position. The successive comparison method is similarly less appropriate than Nickerson's stimulus categorization method, although Grill (1971) describes the former simply as a modification of the latter. The critical difference is that with the successive comparison method, the criterion changes on every trial, a procedure which may encourage a serial mode of processing at the decision level.

A final example of the need to examine the component processes involved in the performance of a given task comes from the experiment of Hawkins (1969). Using a modified version of the Egeth task, Hawkins found that on superficial analysis his "different" results supported a serial interpretation. By means of an elegant examination of the distribution of "different" RTs, he showed that the serial interpretation could, in fact, be rejected. His results were consistent with a self-terminating parallel model, in which the time to process a particular dimension covaries with the average time to process the other dimensions (a property described as "correlated criteria"). Evidence for this property was derived from another experiment, in which each pair of stimuli contained only one dimension, but each block of trials contained stimulus pairs varying on different dimensions. Hawkins assumed that because the task involved only unidimensional stimuli, a serial explanation of the findings of this latter experiment was ruled out. However, this ignores the cybernetic problem of deciding for each stimulus presented which is the relevant dimension. Thus, a process which examined dimensions serially and had as output "same," "different," and "irrelevant" might be able to account for the difference in reaction times between groups. (A third interpretation of this Hawkins result would be in terms of a limited-power parallel model.)

The aim of the present investigation is to test the potentiality for perceptually parallel processing of stimulus dimensions by using a more microscopic analysis, simple but independent dimensions, conjunctively defined response categories, and a single stimulus method. Our first experiment used the Nickerson design but, despite more microscopic analysis, produced results as equivocal as in his comparable experiment. In the second experiment, therefore, the task was simplified for the $S$, while the final experiment combined the two designs. The last two experiments enabled us to make relatively clear-cut inferences about a S's processing in the single-stimulus situation.

\section{EXPERIMENT I}

This experiment was a replication of Nickerson's (1967b) conjunctive condition, using stimuli varying on only two dimensions and with the results analyzed more microscopically. The results of the first experimental session were compared with the overall results, averaged over three sessions, since several Es (e.g., Marcel, 1969; Corcoran, 1971; Grill, 1971) have found changes in the pattern of results with extended practice on the task.

\section{Method}

\section{Apparatus}

The stimuli were presented in a Cambridge tachistoscope. They were drawn with fiber-tip pen on white index cards. On each trial, $E$ inserted a new stimulus card and reset the timer, the click of the reset button acting as a warning signal to $S$. When he was ready, $S$ initiated the stimulus presentation by operating a foot pedal which simultaneously started the timer. The stimulus remained on until $S$ released the foot pedal, after having made his response. Pre- and postexposure fields were white and of approximately the same luminance as the figure surround. The interstimulus interval was approximately $5 \mathrm{sec}$.

The Ss sat with their arms resting on a table and index fingers resting on two plastic keys, which activated microswitches. Depression of either key stopped the timer. which measured the RT to the nearest $0.01 \mathrm{sec}$. All reaction times and responses were recorded by $E$.

\section{Subjects}

Six Ss were used. They were all undergraduate students at University College London who had volunteered to participate. They were each paid $\mathfrak{E} 1$. 
Table 1

Experiment I: Stimuli Grouped into Categories and Corresponding Responses

\begin{tabular}{lllll}
\hline & & \multicolumn{3}{c}{$\begin{array}{c}\text { Experimental Condition } \\
\text { (Relevant Dimension) }\end{array}$} \\
\cline { 3 - 5 } \multicolumn{1}{c}{ Stimulus } & $\begin{array}{l}\text { Stimulus } \\
\text { Category }\end{array}$ & $\begin{array}{l}\text { Shape and } \\
\text { Color }\end{array}$ & Shape & Color \\
\hline $\begin{array}{l}\text { Red Cross } \\
\text { Red Triangle } \\
\text { Yellow Cross }\end{array}$ & S $-\mathrm{C}-$ & No & No & No \\
$\begin{array}{l}\text { Yellow Triangle } \\
\begin{array}{l}\text { Orange Cross } \\
\text { Orange Triangle }\end{array}\end{array}$ & S-C+ & No & No & Yes \\
$\begin{array}{l}\text { Red Circle } \\
\text { Yellow Circle }\end{array}$ & S $+\mathrm{C}-$ & No & Yes & No \\
Orange Circle & $\mathrm{S}+\mathrm{C}+$ & Yes & Yes & Yes \\
\hline
\end{tabular}

\section{Task and Stimuli}

The stimuli were simple geometrical figures which varied along the dimensions of shape and color. Three values on each dimension were used: SHAPE-circle, triangle, cross; COLOR-orange, red. yellow. The sizes of the stimuli were: circle. 0.4 in. (diam); triangle, $0.4 \mathrm{in}$. (height) $\times 0.5 \mathrm{in}$. (base); cross, 0.4 in. (length of arms) $\times 0.1 \mathrm{in}$. (width of arms). The colors were: orange, 5,940 \& (Munsell 2.5YR 6/16); red, 6,140 A (Munsell 5R 5/12); yellow, 5,790 \& (2.5Y 8/16).

All nine possible combinations of these values were used. "Circle" and "orange" were designated as positive (or target) values.

When only one dimension was relevant (Condition S, shape

Table 2

Experiment I: Results Expressed in Form Used by Nickerson (1967b) (RTs in Milliseconds)

\begin{tabular}{cccc}
\hline $\begin{array}{c}\text { Number of } \\
\begin{array}{c}\text { Dimensions (f) } \\
\text { Differing from } \\
\text { the Target }\end{array}\end{array}$ & $\begin{array}{c}\text { Number of Dimensions } \\
\text { Relevant (r) }\end{array}$ & \\
\cline { 2 - 3 } & 2 & 1 & Response \\
\hline 2 & 407 & - & No \\
1 & 460 & 438 & No \\
0 & 407 & 410 & Yes \\
Mean RT & 425 & 424 & \\
\hline
\end{tabular}

Table 3

Experiment I: Detailed Results

\begin{tabular}{llccc}
\hline \multirow{2}{*}{$\begin{array}{l}\text { Stimulus } \\
\text { Category }\end{array}$} & \multicolumn{3}{c}{ Stimulus } & \multicolumn{3}{c}{ Experimental Condition } \\
\cline { 4 - 5 } & SC & S & C \\
\hline \multirow{3}{*}{ S-C -} & Red Triangle & 402 & 395 & 429 \\
& Yellow Triangle & 410 & 405 & 460 \\
& Red Cross & 392 & 401 & 431 \\
S-C+ & Yellow Cross & 418 & 424 & 457 \\
& Orange Triangle & 417 & 395 & 441 \\
S+C- & Orange Cross & 428 & 402 & 447 \\
S+C+ & Red Circle & 443 & 377 & 445 \\
& Yellow Circle & 464 & 407 & 475 \\
& Orange Circle & 407 & 371 & 423 \\
& Error Rates & $3.4 \%$ & $2.4 \%$ & $5.6 \%$ \\
\hline
\end{tabular}

Note-RTs for "Yes" responses are in italics. relevant, or Condition $C$. color relevant), the $S$ was instructed to make a "yes" response if the stimulus possessed the positive value (S+. circle. or $\mathrm{C}+$, orange) on that dimension: he made a "no" response if the stimulus possessed either of the negative values ( $\mathrm{S}-$, triangle or cross; $\mathrm{C}_{-}$. red or yellow). Ss were instructed to ignore the irrelevant dimension in these conditions.

When both dimensions were relevant (Condition SC. shape and color relevant), Ss made a "ves" response only to the stimulus that possessed both positive values (i.e.. $\mathrm{S}^{+} \mathrm{C}^{+}$, orange circle).

The nine stimuli and corresponding responses in each condition are shown in Table 1.

\section{Procedure}

Each experimental condition consisted of 36 trials ( 12 "yes." 24 "no"). Within each condition, each possible "no" stimulus and each possible "yes" stimulus occurred equally often.

The Ss attended for four sessions: one practice and three experimental sessions. The practice session consisted of familiarization with the apparatus, in which Ss made choice responses to letters of the alphabet, followed by two practice blocks of 12 trials under each condition. The experimental sessions were divided into two halves, with a 2 -min rest break in the middle. Each condition was presented once in each half of the session, the order of conditions over the three sessions forming two Latin squares. Ss were given six practice trials in each condition before it was presented for the first time each session. Instructions regarding condition were given before each block of trials. A card showing the nine stimuli was displayed next to the tachistoscope throughout the experiment.

Half the Ss used their preferred hands for the "yes" and half used them for the "no" response. They were instructed to respond as quickly and accurately as possible.

\section{Results and Discussion}

Table 2 shows the results expressed in the form of Nickerson (1967b) and Marcel (1969). Following Nickerson, RTs are given in terms of medians.

On an exhaustive model, either parallel or serial, RTs should be much slower, on average, when two dimensions are relevant than when only one dimension is relevant. This is clearly not the case in Nickerson's data or in Table 2, which shows that the mean two-dimensional RT $(425 \mathrm{msec})$ is as fast as the mean one-dimensional RT ( $424 \mathrm{msec}$ ).

In the following discussion, we are considering only those aspects of the results which illustrate the limitations of this method of analysis. The comparisons considered all involve "no" responses.

If the stimulus differs from the target on one dimension only $(f=1)$, Ss are significantly faster when only the "critical" dimension is relevant $(r=1)$ [ $t(5)=$ $2.33, p<.05]$. As described above, this seems to refute a parallel model and to support a serial model, as it appears that in the slower condition $(r=2)$, the "noncritical" dimension is sometimes processed first.

However, Table 3 gives the results in a more detailed form, and it is possible to show that this conclusion is not warranted. In particular, in Table 3 , the stimuli which differ from the target on one dimension have been separated into those which differ in shape (S-C+ stimuli) and those which differ in color $(\mathrm{S}+\mathrm{C}-$ stimuli), 
so that it is possible to specify the serial model that is consistent with the result described above. (i) For the $\mathrm{S}-\mathrm{C}+$ stimuli, RTs are significantly slower when both dimensions are relevant (Condition SC) than when only the "critical" dimension (shape) is relevant (Condition $\mathrm{S}$ ) $[\mathrm{t}(5)=3.27, \mathrm{p}<.025]$. This result implies that the "noncritical" dimension (color) has been processed first on at least some of the trials in the two-dimensional condition. (ii) For the $\mathrm{S}+\mathrm{C}-$ stimuli, there is no significant difference between the RT when both dimensions are relevant (Condition SC) and when only the "critical" dimension (color) is relevant (Condition $\mathrm{C}$ ) $[\mathrm{t}(5)=0.98, \mathrm{p}>.15]$. This result implies that the critical dimension (color) was always processed first when both dimensions were relevant. Thus, Results $i$ and ii together mean that the significant difference shown in Table 2 can be explained on a serial model only if color is processed first.

However, this detailed conclusion is not consistent with the results for the S-C-stimuli, which differ from the target on both dimensions, and which can therefore be identified on the basis of either dimension. A two-way ANOVA of the four $\mathrm{S}-\mathrm{C}$ - stimuli in the three experimental conditions shows that in the two-dimensional condition these stimuli are identified as rapidly as when shape alone is relevant (Condition S) $[\mathrm{F}(1,6)<1]$ and significantly faster than when color is relevant (Condition $\mathrm{C}$ ) $[\mathrm{F}(1 ., 6)=78.5, \mathrm{p}<.005)$. This result is consistent with a serial model only if shape is processed first.

Thus, one comparison implies that the dimension processed first must be color, but the other comparison implies that it must be shape. Hence, although individual comparisons in the data suggest a serial explanation, it is difficult to see how the total pattern of results could be accounted for in that way.

It, therefore, appears that, even considering only the negative stimuli, it is necessary to complicate the original model. Three tentative possibilities are presented in increasing order of plausibility:

(1) The difficulties encountered by a serial model have been described above. However, a serial model, in which shape is normally processed first, could account for all the results if it is assumed that Ss have difficulty in altering the order in which they process dimensions during the course of a session. This would mean that shape is frequently processed first, not only when both dimensions are relevant (Condition SC), but also when color only is relevant (Condition C). This would explain the lack of difference in RTs between the SC and $\mathrm{C}$ conditions for the $\mathrm{S}+\mathrm{C}-$ stimuli (see Result ii above).

(2) The results could possibly be accounted for by a "limited-power" parallel model (as described by Corcoran, 1971), in which the parallel processing of two dimensions is less efficient than the processing of either dimension alone. However. in this case, the model would have difficulty in explaining why the two $\mathrm{S}+\mathrm{C}$ - stimuli (red circle, yellow circle) are processed as rapidly when two dimensicns are relevant (Condition SC) as when color is relevani (Condition C) (Result ii above).

(3) The only result incompatible with an unlimited parallel model is that the $\mathrm{S}-\mathrm{C}+$ stimuli (orange triangle, orange cross) are processed more slowly when both dimensions are relevant (Condition SC) than when shape is relevant (Condition $S$ ). If it is assumed that $\mathrm{Ss}$ cannot totally ignore the results of color processing when the decision has to be made on the basis of shape, then this result could be explained in terms of response competition, since the values on the two-component dimensions, $\mathrm{S}-, \mathrm{C}_{+}$, would be associated with different responses. There is some evidence in the results for the "yes" stimuli of interference from the irrelevant dimension (color) when shape is relevant (Condition S). The circle ( $\mathrm{S}+$ ) was processed more rapidly when paired with the positive color value (orange) than with either of the negative values (red or yellow), even though color was irrelevant $[F(2,10)=28.3, p<.005]$. This explanation is made more plausible by evidence in the literature that Ss cannot "filter out" color when it is an irrelevant dimension (Egeth \& Pachella, 1969; Morgan \& Alluisi, 1967).

\section{EXPERIMENT II}

This last possibility suggests that it would be better to test the models in a situation in which response competition cannot operate. This would involve excluding all stimuli for which the two component dimensions are associated with opposite responses (i.e., $\mathrm{S}+\mathrm{C}-$ and $\mathrm{S}-\mathrm{C}+$ stimuli). It would seem to be very difficult to distinguish between the models in this situation, since the remaining stimulus categories $(\mathrm{S}+\mathrm{C}+$, $\mathrm{S}-\mathrm{C}-$ ) $\mathrm{can}$ both be identified on the basis of either dimension alone. Therefore, both self-terminating serial and parallel models predict that, for all stimuli, when two dimensions are relevant, the response will be at least as fast as the fastest component dimension.

One method is to use the concept of "redundancy gain," as in the work of Biederman and Checkosky (1970). If the variance of the time to perceptually process each individual dimension is not zero and the distributions overlap, then a parallel model should predict a faster mean RT when both dimensions are relevant than when only one is relevant. However, the difference would be expected to be small. As pointed out earlier, in their experiment there is the problem that their Ss might conceivably have collapsed the two dimensions into a single dimension for processing.

Another way to differentiate the models is to vary the difficulty of discrimination within each dimension. Suppose that there are two negative values on each dimension, such that one value $(-E)$ is easy to discriminate from the positive value and the other value (-D) is difficult to discriminate. The three values on each dimension can then be referred to as $t_{1},-E_{1}$. $-D_{1}: t_{2},-E_{2},-D_{2}:$ where the suffixes 1.2 denote the 
Table 4

Experiment II: Experimental Conditions and Stimuli

\begin{tabular}{|c|c|c|c|c|c|}
\hline Relevant Dimension & Instruction & & Stimuli & & $\begin{array}{l}\text { Irrelevant } \\
\text { Dimension }\end{array}$ \\
\hline Shape (S) & Is it a square? & $\begin{array}{l}\text { Square } \\
t_{1}\end{array}$ & $\begin{array}{l}\text { Rectangle } \\
-D_{1}\end{array}$ & $\begin{array}{l}\text { Triangle } \\
-E_{1}\end{array}$ & Green \\
\hline Color (C) & Is it red? & $\begin{array}{l}\text { Red } \\
t_{2}\end{array}$ & $\begin{array}{l}\text { Orange } \\
-D_{2}\end{array}$ & $\begin{array}{l}\text { Blue } \\
-E_{2}\end{array}$ & Circle \\
\hline Shape and Color (SC) & Is it a red square? & $\begin{array}{l}\text { Red Square } \\
\left(+_{1},+_{2}\right)\end{array}$ & $\begin{array}{l}\text { Orange Triangle } \\
\left(-E_{1},-D_{2}\right)\end{array}$ & $\begin{array}{l}\text { Blue Rectangle } \\
\left(-D_{1},-E_{2}\right)\end{array}$ & - \\
\hline \multicolumn{2}{|c|}{ Correct Response } & Yes & No & No & \\
\hline
\end{tabular}

two dimensions and + denotes the positive value. Consider the condition when two dimensions are relevant. The parallel model predicts that the two negative stimuli $\left(-E_{1},-D_{2}\right)$ and $\left(-D_{1},-E_{2}\right)$ will be discriminable at least as quickly as $-\mathrm{E}_{1},-\mathrm{E}_{2}$, respectively, from the positive stimulus $\left(t_{1}, t_{2}\right)$. However. on any serial model, the difficult dimension must be processed first on some trials, since it cannot be known in advance which dimension will be the easy one for a particular stimulus. Therefore, one or both of the negative stimuli $\left(-\mathrm{E}_{1},-\mathrm{D}_{2}\right)$ and $\left(-\mathrm{D}_{1},-\mathrm{E}_{2}\right)$ will be processed more slowly than $-E_{1},-E_{2}$, respectively.

If only the three stimuli $\left(+_{1},+_{2}\right),\left(-E_{1},-D_{2}\right),\left(-D_{1}\right.$, $-E_{2}$ ) are used, then this design has the additional advantage that it allows all comparisons to be made across stimuli occurring with equal frequency, which is not possible with the Nickerson design.

\section{Method}

\section{Task and Stimuli}

The experimental conditions, instructions, and stimulus values used are shown in Table 4. The sizes of the stimuli were: square, 0.4 in. (side); triangle, 0.4 in. (height) $\times 0.5$ in. (base); rectangle, 0.4 in. (height) $\times 0.5$ in. (width); circle, 0.4 in. (diam). The colors, expressed in wavelengths and Munsell notation were: red, $6,140 \AA(5 R 5 / 12) ;$ orange, $6,000 \AA(10 R 5 / 14) ;$ blue, $4,000 \AA$ (7.5PB 3/12); green, 5,510 \& (10GY 6/10).

In both single-dimension relevant conditions $(S, C)$, the irrelevant dimension was held constant, taking a value which did not occur elsewhere in the experiment.

\section{Subjects}

Six undergraduate students of University College London volunteered to participate in the experiment. They were each paid $\mathcal{L} 1$ for their services.

\section{Procedure}

Each experimental condition consisted of a block of 24 trials. "Yes" and "no" responses occurred equally of ten, as did each of the "no" stimuli within each condition.

The nature of the stimuli was carefully explained to the Ss. That is, the instructions stated explicitly that in the two-dimensional relevant condition (SC) they could make their decision on the basis of either dimension alone.

In all other respects, the method was as for Experiment $I$.

\section{Results and Discussion}

Table 5 shows the median response times averaged across the six Ss.

(1) The time for "orange triangle" in Condition C is not significantly slower than that for "triangle" (Condition $S$ ) $[t(5)=0.99, p>.2]$, both times being significantly faster than the time for "orange" (Condition C) $[t(5)=7.99, p<.001]$. Also, the time for "blue rectangle" (Condition $\mathrm{SC}$ ) is not significantly slower than that for "blue" (Condition C) [t $(5)=1.83$. $p>.1]$, while both times are significantly faster than the time for "rectangle" (Condition $S$ ) $[\mathrm{t}(5)=4.65$, $p<.01]$. These results hold and are significant $(p<.025)$ for the individual scores of all six Ss.

Thus, as predicted by the parallel model, both the

Table 5

Experiment II: Results for Each Session and for Data Pooled Over Three Sessions

\begin{tabular}{|c|c|c|c|c|c|c|c|c|c|}
\hline \multirow[b]{3}{*}{ Stimulus } & \multicolumn{3}{|c|}{ “No" Response } & \multicolumn{3}{|c|}{$\begin{array}{c}\text { "No" Response } \\
\text { Experimental Condition }\end{array}$} & \multicolumn{3}{|c|}{ "Yes" Response } \\
\hline & \multirow{2}{*}{$\begin{array}{c}\text { SC } \\
\text { Orange } \\
\text { Triangle }\end{array}$} & S & $\mathrm{C}$ & \multirow{2}{*}{$\begin{array}{c}\mathrm{x} \\
\text { Blue } \\
\text { Rectangle }\end{array}$} & $\mathbf{S}$ & C & SC & $\mathrm{S}$ & $\mathrm{C}$ \\
\hline & & Triangle & Orange & & Rectangle & Blue & $\begin{array}{c}\text { Red } \\
\text { Square }\end{array}$ & Square & Red \\
\hline Session $\begin{array}{l}1 \\
2 \\
3\end{array}$ & $\begin{array}{l}421 \\
413 \\
423\end{array}$ & $\begin{array}{l}416 \\
401 \\
406\end{array}$ & $\begin{array}{l}504 \\
484 \\
474\end{array}$ & $\begin{array}{l}405 \\
403 \\
396\end{array}$ & $\begin{array}{l}488 \\
468 \\
455\end{array}$ & $\begin{array}{l}417 \\
419 \\
410\end{array}$ & $\begin{array}{l}388 \\
375 \\
378\end{array}$ & $\begin{array}{l}419 \\
398 \\
406\end{array}$ & $\begin{array}{l}433 \\
418 \\
429\end{array}$ \\
\hline Pooled Data & 418 & 412 & 479 & 402 & 472 & 418 & 379 & 405 & 429 \\
\hline & & rror rates & each & on were & $9 \%(\mathrm{SC}), 5$ & (S), & (C). & & \\
\hline
\end{tabular}


negative stimuli ("orange triangle" and "blue rectangle") were processed as quickly as their easy dimension. This result is incompatible with any sort of serial model. since the $S$ has no way of knowing which dimension will be the easy one for the next stimulus. There is also no support for a "limited-power" parallel model since the combined two-dimensional time is as fast as the combined faster single-dimension times $(820 \mathrm{msec}$ compared with $830 \mathrm{msec}$ ).

(2) The "yes" results are also consistent with an unlimited parallel model and inconsistent with a serial one. "Red square" (Condition SC) is faster than both "red" (Condition C) and "square" (Condition S). [Red square $<\min$ (red. square), not significant $-\mathrm{t}(5)=1.31$, $p>.1$-but the difference is significant $-p<.01-$ for the individual results of three of the six Ss.]

(3) The results show no tendency for any qualitative change to occur with practice, except possibly for the difficult single-dimension stimuli to become faster.

It is conceivable that the small number of different stimuli in each condition allowed templates to be formed for each stimulus. However, a similar experiment. adding a yellow pentagon and a turquoise diamond, showed almost the same results; the two additional stimuli were discriminated insignificantly faster than their respective fastest component dimension.

\section{EXPERIMENT III}

When any possibility of response competition was excluded by the experimental design, the results showed clear support for a parallel model. This also provides indirect support for the third interpretation of Experiment I. namely. that the results represent deviations from a parallel model, caused by response competition. On the other hand, it is possible that Experiment II involved an especially simple situation for the S. and that "parallel" processing is restricted to such situations. However, this method of using different difficulties of discrimination can be adapted to the Nickerson (1967b) situation, to provide direct evidence on this question. If the stimulus values used in Experiment II are used in the situation of Experiment I (i.e., including all nine possible combinations of the stimulus values), then the specific predictions tested in Experiment II are still valid. These predictions concern the RTs to the double negative stimuli $(\mathrm{S}-\mathrm{C}-$ ) in all three conditions; the predictions regarding the stimuli with competing stimulus values $(\mathrm{S}-\mathrm{C}+\mathrm{S}+\mathrm{C}-$ ), which were discussed in relation to Experiment $\mathrm{I}$, provide a further test of the models.

\section{Method}

\section{Subjects}

Ten undergraduate students of University College London
Table 6

Experiment III: Data Pooled Over Three Sessions

\begin{tabular}{|c|c|c|c|c|}
\hline \multirow{2}{*}{$\begin{array}{l}\text { Stimulus } \\
\text { Category }\end{array}$} & \multirow[b]{2}{*}{ Stimulus } & \multicolumn{3}{|c|}{ Experimental Condition } \\
\hline & & $\mathrm{SC}$ & $S$ & $\mathrm{C}$ \\
\hline $\mathrm{S}-\mathrm{C}-$ & $\begin{array}{l}\text { Orange Triangle } \\
\text { Blue Rectangle } \\
\text { Orange Rectangle } \\
\text { Blue Triangle }\end{array}$ & $\begin{array}{l}474 \\
434 \\
488 \\
428\end{array}$ & $\begin{array}{l}457 \\
491 \\
494 \\
442\end{array}$ & $\begin{array}{l}599 \\
449 \\
554 \\
441\end{array}$ \\
\hline $\mathrm{S}-\mathrm{C}+$ & $\begin{array}{l}\text { Red Triangle } \\
\text { Red Rectangle }\end{array}$ & $\begin{array}{l}469 \\
508\end{array}$ & $\begin{array}{l}458 \\
509\end{array}$ & $\begin{array}{l}524 \\
514\end{array}$ \\
\hline $\mathrm{S}+\mathrm{C}-$ & $\begin{array}{l}\text { Orange Square } \\
\text { Blue Square }\end{array}$ & $\begin{array}{l}569 \\
439\end{array}$ & $\begin{array}{l}480 \\
498\end{array}$ & $\begin{array}{l}567 \\
445\end{array}$ \\
\hline $\mathrm{S}+\mathrm{C}+$ & Red Square & 502 & 472 & 502 \\
\hline & Error Rates & $1.25 \%$ & $1.25 \%$ & $2.2 \%$ \\
\hline
\end{tabular}

Note-RTs for "Yes" responses are in italics.

volunteered to participate in the experiment, for which they were paid $\mathfrak{1} 1$.

\section{Task and Stimuli}

The same stimulus values were used as in Experiment II, but all nine possible combinations of these values were included. Ss were instructed to ignore the irrelevant dimensions in single-dimension conditions.

In all other respects the method was as for Experiment $I$.

\section{Results and Discussion}

Table 6 shows the median response times for the pooled data of three sessions, averaged over the $10 \mathrm{Ss}$. The following conclusions can be drawn:

(1) The critical comparisons regarding the $\mathrm{S}-\mathrm{C}-$ stimuli, which were made in Experiment II, can also be made here: (i) there is no significant difference in RT to "orange triangle" between Condition $\mathrm{SC}$, in which both dimensions are relevant, and Condition $S$, in which only the easy dimension (shape) is relevant $[\mathrm{t}(9)=1.61$, $\mathrm{p}>$.1], both times being significantly faster than the corresponding RT when color is relevant (Condition C) $[\mathrm{t}(9)=4.19, \mathrm{p}<.01]$. (ii) There is no significant difference in RT to "blue rectangle" when both dimensions are relevant (Condition SC) and when the easy dimension (color) is relevant (Condition $\mathrm{C})[\mathrm{t}(9)=$ $1.87, p>.05]$. Both these times are significantly faster than the corresponding RT when shape is relevant (Condition S) $[\mathrm{t}(9)=4.36, \mathrm{p}<.01]$.

Thus, as in Experiment II, both these stimuli were identified as rapidly as their easy dimension. These two results hold and are significant $(p<.05)$ for the individual results of 8 out of the $10 \mathrm{Ss}$, and thus strongly support a parallel model. (The combined two-dimensional condition time is $908 \mathrm{msec}$ compared with the fastest single-dimension time of $906 \mathrm{msec}$.) The serial model is therefore made very implausible. as is a "limited-power" parallel model.

(2) The results for the other two double negative (S-C-) stimuli ("orange rectangle" and "blue triangle") 
Table 7

Experiment III: Results for First Session Only

\begin{tabular}{llcc}
\hline & \multicolumn{3}{c}{ Experimental Condition } \\
\cline { 2 - 4 } \multicolumn{1}{c}{ Stimulus } & SC & S & $\mathrm{C}$ \\
\hline Orange Triangle & 501 & 477 & 645 \\
Blue Rectangle & $\mathbf{4 6 0}$ & 503 & 456 \\
Orange Rectangle & 514 & 501 & 587 \\
Blue Triangle & 449 & 472 & 469 \\
Red Triangle & 502 & 484 & 561 \\
Red Rectangle & 526 & 544 & 535 \\
Orange Square & 580 & 516 & 593 \\
Blue Square & 444 & 520 & 466 \\
Red Square & 520 & 466 & 474 \\
\hline
\end{tabular}

Note-RTs for "Yes" responses are in italics.

are clearly compatible with the parallel model, since they were both processed as quickly as their faster component dimension: (i) For "orange rectangle," there is no significant difference between RTs when both dimensions are relevant (Condition SC) and when the fastest single dimension (shape) is relevant (Condition $\mathrm{S}$ ) $[t(9)=1.58, p>.05]$; both these times are significantly faster than the slower single-dimension condition (C) $[t(9)=7.33, p<.001]$. (ii) For "blue triangle," RTs in the both-dimensions-relevant condition (SC) were insignificantly faster than the faster of the single-dimension-relevant conditions, the latter being defined separately for each $\mathrm{S}[\mathrm{t}(9)=0.67, \mathrm{p}>.25]$.

(3) The results for the stimuli with competing stimulus values ( $\mathrm{S}-\mathrm{C}+$ and $\mathrm{S}+\mathrm{C}-$ stimuli) also support a parallel model. All four of these stimuli were processed as rapidly when both dimensions were relevant (Condition SC) as when the "critical" single dimension $(\mathrm{S}, \mathrm{C}$, respectively) was relevant. Red triangle, $\mathrm{t}(9)=$ 0.69 ; red rectangle, $t(9)=0.02$; orange square, $t(9)=$ -0.06 ; blue square, $\mathrm{t}(9)=-1.64 ; \mathrm{p}>.05$ for all stimuli. Since Ss have no way of knowing which dimension will be the "critical" one on the next trial, this result cannot be compatible with a serial model which predicts that at least one of the stimulus categories ( $\mathrm{S}-\mathrm{C}+$ or $\mathrm{S}+\mathrm{C}-$ ) should be significantly slower when both dimensions are relevant.

(4) The same conclusions can be drawn from the results of the first experimental session (see Table 7), showing that there is no qualitative change of strategy with practice.

The interpretation of these experiments is based on comparisons made across different experimental conditions. There would appear to be no a priori reason why such comparisons should not be valid. However, it is conceivable that, in this and the previous experiment, the existence of more difficult discriminations in single-dimension conditions might lead Ss to adopt a more rigid criterion in these conditions. This is the "correlated criteria" property suggested by Hawkins (1969), but as discussed earlier not, in our opinion, clearly demonstrated by him. Errors are too few
(Experiment II, 5.1\%; Experiment III, 1.6\%) to make an estimate of criterion shift. However, there are a number of reasons why a serial explanation cannot be valid, even if such an effect is considered. (i) If the stimuli in the two-dimensional relevant condition (SC) have been processed misleadingly fast, then this should also have affected the "yes" responses. However. RT to the positive stimulus (red square) in Condition $\mathrm{SC}$ is equal to the slower of the two single-dimension-condition times (Condition C), as the parallel model predicts. (ii) If the results of the two-dimensions-relevant condition (SC) are considered alone (see Fig. 1), it can be shown that they are not compatible with a serial model. The similarity between all the RTs to blue stimuli, and the fact that they are significantly faster than all other stimuli, indicates that color must be processed first in any serial process. Yet, if this is the case, the orange stimuli should also all be processed equally rapidly, and they should all be faster than the red negative stimuli, neither of which is the case.

It is just conceivable that Ss adopt a more complex serial strategy which involves switching from one dimension to the other, so that the easy values on each dimension are tested first and the difficult values are tested only if a decision has not already been reached. Since "orange square" shows the slowest RT, "orange" must be the attribute normally processed last; similarly "blue" must be processed first. The only possible process of this kind would be: "Is it blue?" "If no, is it a triangle?" "If no, is it a rectangle?" "If no, is it orange?" This would mean that "orange rectangle" and "red rectangle" would be processed equally quickly. However, there is a significant difference between their response times (see Fig. 1), which shows that such a strategy has not been unanimously adopted. Analysis of the results of individual Ss shows that, for $7 / 10 \mathrm{Ss}$, no serial strategy, however complex, can be arranged to fit their results.

(iii) Finally, considering the negative RTs, it is most implausible that RTs in Condition SC (two dimensions relevant) have been "speeded up." As described above,

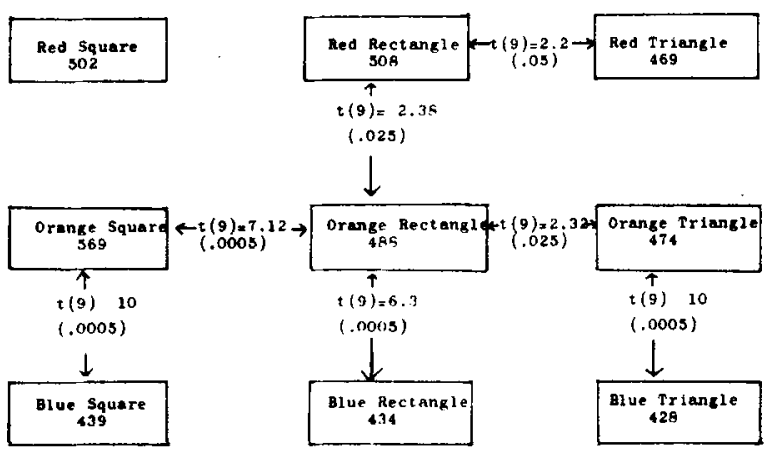

Fig. 1. The results for the two-dimensional condition (SC) in Experiment III, showing significant differences in processing times between stimuli (significance levels are shown in brackets). 
the only possible serial strategy is one in which "blue" is processed first and "orange" is processed last. Thus, a serial model predicts that the time to process "blue square" is the same whether one or two dimensions are relevant (i.e., Conditions $\mathrm{C}, \mathrm{SC}$ ). On the other hand, the time to process "orange square" when both dimensions are relevant (Condition SC) must be equal to the time to process "blue," "triangle," "rectangle," and "orange." The RT to "orange square" should therefore be much greater when both dimensions are relevant (Condition SC) than when color is relevant (Condition C). In fact, the RTs do not differ significantly (see Result 3 above), as predicted on a parallel model.

Hence, the data seem to overwhelmingly support an unlimited parallel model rather than a serial model.

\section{GENERAL DISCUSSION AND CONCLUSIONS}

The results of these experiments strongly suggest that parallel processing can occur in the identification of single multidimensional stimuli. Since these results were obtained even with unpracticed Ss, they also provide evidence against the idea that practice is necessary for parallel processing to occur (e.g., Marcel, 1969; Corcoran, 1971; Grill, 1971). This agrees with the conclusions of Hawkins (1969) and Downing and Gossman (1970), using the Egeth design. Given that the results of Posner and Boies (1971), using a very different divided attention situation, support a similar conclusion, it seems plausible that the normal mode of perceptual processing is parallel, given relatively easily discriminated stimuli.

Other experiments of the present type (e.g., Marcel, 1969 , and possibly Nickerson, 1967b) do not show these parallel characteristics, at least not in the early stages of practice. There are two possible explanations of this which do not conflict with the notion of parallel processing at the perceptual level.

Firstly, it is possible that the serial appearance of Nickerson's and Marcel's results could be due to an artifact produced by averaging across dimensions. The analysis of Experiment $I$ shows that such artifacts can occur, and this is most likely if Ss have difficulty in ignoring one of the dimensions (as in Experiment I), or if there is a great difference in discriminability between dimensions, as is certainly the case for Marcel, though Nickerson gives no information on this. If the perceptual process operates in parallel, and if practice has a greater effect on more difficult discriminations, as in our experiments, then the pattern of response times would change in the way that Marcel finds.

Secondly, the seemingly serial results of Nickerson and Marcel might be due to the greater cognitive load placed on the decision process by their task (three dimensions instead of two). The change in the pattern of results from "serial" to "parallel" processing with extended practice, which Marcel finds. would therefore be due to a change of strategy by the S. In Marcel's experiment, Ss performed both disjunctive and conjunctive tasks. They would therefore be likely to adopt a very cautious decision strategy in the early stages of practice.

This second explanation, like some of the considerations raised in the Introduction, depends upon differentiating between perceptual and decision mechanisms. Such a differentiation fits a number of different theories [e.g., Miller, Galanter, \& Pribram, 1960 (image/plan); Shallice, 1972 (perceptual system/action systems]. On such an approach, the operation of the perceptual mechanism, whose output selects which response-control system is to be activated, is relatively unaffected by task instructions or the S's strategy. It corresponds more to a passive view of the perceptual process than to an active one.

Several investigators have found that different stimulus dimensions produce different patterns of results (e.g., Garner \& Felfoldy, 1970; Egeth \& Pachella, 1969). The nature of the dimensions used could affect both the S's ability for perceptual processing in parallel and also his decision strategy. Lockhead (1966) and Garner and Felfoldy (1970) have used the concept of "integrality" of dimensions to account for these differences. However, neither the criterion for integrality nor the relationship between integrality and parallel processing seem to be clear. Thus, Lockhead (1966) defines as "integral," "all of those dimensions which can be presented simultaneously and in the same place, while maintaining psychological independence." The two dimensions used in the present experiments certainly satisfy this criterion. However, these dimensions do not show a "redundancy gain" in speed, which is the criterion of integrality used by Garner and Felfoldy (1970).

It might seem that, in general, the lack of a redundancy gain would imply that the underlying processes are not in parallel, and this inference seems to be made by Felfoldy and Garner (1971). This is not necessarily the case, as the times to process the individual dimensions can be very different, as in our experiments. In fact, in the present experiments, which do not show a clear redundancy gain, the results are consistent only with a parallel model. This is because several stimuli appear in a block of trials, and the fastest component dimension cannot be the same for all these stimuli.

Thus, "redundancy gain" appears to be an unreliable criterion upon which to assess integrality. However, it remains possible that an improved concept of integrality could be related to dimensions that are perceptually processed both independently and in parallel, as in Morton's (1969) "decision combination." The alternative possibility is that integrality should refer to dimensions for which, in signal detection terms, $d^{\prime}$ values are summed before a perceptual decision is made, as perhaps for Garner and Felfoldy"s "value" and "chroma" of single Munsell chips-Morton's (1969) 
"evidence-combination." In the latter case, the question of parallel processing, as discussed in this paper, will probably prove to be an independent question.

\section{REFERENCES}

Biederman, I.. \& Checkosky, S. F. Processing redundant information. Journal of Experimental Psychology, 1970, 83, 486-490.

Corcoran. D. W. J. Pattern recognition. Harmondsworth: Penguin. 1971

Downing, B. D.. \& Gossman, J. R. Parallel processing of multidimensional stimuli. Perception \& Psychophysics, 1970, $8.57-60$.

Egeth. H. Parallel versus serial processes in multidimensional stimulus discrimination. Perception \& Psychophysics, 1966, 1, 245-252.

Egeth, H., \& Pachella. R. Multidimensional stimulus identification. Perception \& Psychophysics, 1969, 5, 341-346.

Felfoldy. G. R., \& Garner, W. R. The effects on speeded classification of implicit and explicit instructions regarding redundant dimensions. Perception \& Psychophysics, 1971, 9 , 289-292.

Garner. W. R., \& Felfoldy, G. R. Integrality of stimulus dimensions in various types of information processing. Cognitive Psychology, 1970, 1, 225-241.

Garner, G. R., \& Morton, J. Perceptual independence, Psychological Bulletin, 1969, 72, 233-259.

Grill. D. P. Variables influencing the mode of processing of complex stimuli. Perception \& Psychophysics, 1971, 10, 51-57.

Hawkins. H. L. Parallel processing in complex visual discrimination. Perception \& Psychophysics, 1969, 5, 56-64.

Lindsay, P. H., Taylor, M. M., \& Forbes, S. M. Attention and multidimensional discrimination. Perception \& Psychophysics, 1968. 4. 113-117.

Lindsay. R. K.. \& Lindsay. J. M. Reaction time and serial versus parallel information processing. Journal of Experimental Psychology, 1966, 71, 294-303.

Lockhead, G. R. Effects of dimensional redundancy on visual discrimination. Journal of Experimental Psychology, 1966, 72, 95-104.
Marcel. A. J. Serial and parallel processing in pattern recognition. Paper read at London Conference of the Experimental Psychology Society, 1969.

Marcel, A. J. Some constraints on sequential and parallel processing, and the limits of attention. Acta Psychologica, $1970,33,77-92$.

Miller. G. A., Galanter, E., \& Pribram, K. H. Plans and the structure of behavior. New York: Holt, 1960.

Morgan, B. B., Jr., \& Alluisi, E. A. Effects of discriminability and irrelevant information on absolute judgments. Perception \& Psychophysics, 1967, 2, 54-58.

Morton, J. The use of correlated stimulus information in card sorting. Perception \& Psychophysics, 1969, 5, 374-376.

Neisser, U. Decision-time without reaction time: Experiments in visual scanning. American Journal of Psychology, 1963, 76, 376-385.

Nickerson. R. S. 'Same'-'Different' response times with multiattribute stimulus differences. Perceptual \& Motor Skills, 1967a. 24, 543-554.

Nickerson. R. S. Categorization time with categories defined by disjunctions and conjunctions of stimulus attributes. Journal of Experimental Psychology, 1967b, 73, 211-219.

Posner, M. I., \& Boies, S. J. Components of attention. Psvchological Review, 1971, 28, 391-408.

Rabbitt. P. M. A. Time for analysing stimuli and selecting responses. British Medical Bulletin, 1971, 27, 259-265.

Shallice, T. Dual functions of consciousness. Psychological Review, 1972, 79, 383-393.

Sternberg, S. Retrieval from recent memory: Some reaction time experiments and a search theory. Paper presented to the Psychonomic Society, Bryn Mawr, Pennsylvania, 1963.

Trabasso. T., Rollins. H., \& Shaugnessy, E. Storage and verification stages in processing concepts. Cognitive Psychology, 1971, 2, 239-289.

Treisman, A. M. Strategies and models of selective attention. Psychological Review, 1969, 76, 282-299.

(Received for publication September 3, 1972; revision received November $22,1972$. ) 